\title{
Auger Processes Mediating the Nonresonant Optical Emission from a Semiconductor Quantum Dot Embedded Inside an Optical Cavity
}

Settnes, Mikkel; Nielsen, Per Kær; Lund, Anders Mølbjerg; Mørk, Jesper

Published in:

Physical Review Letters

Link to article, DOI:

10.1103/PhysRevLett.111.067403

Publication date:

2013

Document Version

Publisher's PDF, also known as Version of record

Link back to DTU Orbit

Citation (APA):

Settnes, M., Nielsen, P. K., Lund, A. M., \& Mørk, J. (2013). Auger Processes Mediating the Nonresonant Optical Emission from a Semiconductor Quantum Dot Embedded Inside an Optical Cavity. Physical Review Letters, 111(6), [067403]. https://doi.org/10.1103/PhysRevLett.111.067403

\section{General rights}

Copyright and moral rights for the publications made accessible in the public portal are retained by the authors and/or other copyright owners and it is a condition of accessing publications that users recognise and abide by the legal requirements associated with these rights.

- Users may download and print one copy of any publication from the public portal for the purpose of private study or research.

- You may not further distribute the material or use it for any profit-making activity or commercial gain

- You may freely distribute the URL identifying the publication in the public portal 


\title{
Auger Processes Mediating the Nonresonant Optical Emission from a Semiconductor Quantum Dot Embedded Inside an Optical Cavity
}

\author{
Mikkel Settnes, ${ }^{1,2, *}$ Per Kaer, ${ }^{1}$ Anders Moelbjerg, ${ }^{1}$ and Jesper Mork ${ }^{1}$ \\ ${ }^{1}$ Department of Photonics Engineering, DTU Fotonik, Technical University of Denmark, DK-2800 Kongens Lyngby, Denmark \\ ${ }^{2}$ Department of Micro- and Nanotechnology Engineering, Center for Nanostructured Graphene (CNG), \\ Technical University of Denmark, DK-2800 Kongens Lyngby, Denmark
}

(Received 21 October 2012; published 7 August 2013)

\begin{abstract}
We show that Auger processes involving wetting layer transitions mediate emission from a cavity that is detuned from a quantum dot by even tens of meV. The wetting layer thus acts as a reservoir, which by Coulomb scattering can supply or absorb the energy difference between emitter and cavity. We perform microscopic calculations of the effect treating the wetting layer as a non-Markovian reservoir interacting with the coupled quantum dot-cavity system through Coulomb interactions. Experimentally, cavity feeding has been observed in the asymmetric detuning range of -10 to $+45 \mathrm{meV}$. We show that this asymmetry arises naturally from the quasiequilibrium properties of the wetting layer reservoir. Furthermore, we present numerical calculations of both photoluminescence spectra and photon correlations, demonstrating good qualitative agreement with experiments.
\end{abstract}

DOI: 10.1103/PhysRevLett.111.067403

A semiconductor quantum dot (QD) coupled to an optical cavity, such as a semiconductor micropillar [1-3] or a photonic crystal defect cavity $[4,5]$, has proven an interesting system for realizing cavity quantum electrodynamics (cQED) effects in solid-state systems [6] and a promising platform for future quantum information technologies [7]. Compared to atom-based cQED systems, QD-based systems have unique properties arising from interactions with the solid-state environment. Several experiments demonstrated strong emission from a cavity containing a single $\mathrm{QD}$, even when the QD was strongly detuned from the cavity resonance [8-12]. This strong off-resonant emission was observed in the asymmetric detuning range of $\hbar \Delta \sim$ $-10 \mathrm{meV}$ to $\hbar \Delta \sim+45 \mathrm{meV}[9,13]$, where $\Delta=\omega_{\mathrm{QD}}-$ $\omega_{C}$ is the detuning between the QD exciton line $\left(\omega_{\mathrm{QD}}\right)$ and the cavity frequency $\left(\omega_{C}\right)$, thus greatly exceeding the cavity and emitter linewidths. Experimentally, it was confirmed by photon-correlation measurements that nonresonant cavity feeding is not a feature of the cavity itself, nor is it caused by multiple QDs $[4,5]$.

It is well established that the QD interacts with the semiconductor environment, often accounted for by more or less phenomenological scattering terms. Pure dephasing effects have been shown to enable cavity feeding in systems with small detuning $(|\hbar \Delta|<5 \mathrm{meV})$ [14-16]. In that case, interaction with acoustic phonons plays the major role [17-24], but this interaction cannot explain the cavity feeding observed for larger detunings $(|\hbar \Delta|>5 \mathrm{meV})$. Longitudinal optical (LO) phonons may lead to interactions in a larger spectral range, but the discrete nature of LO phonons gives rise to characteristic discrete spectral peaks [25], at variance with the experimental observation of a broad emission background. The low temperature further limits the role of LO phonons [26,27].
PACS numbers: 78.67.Hc, 42.50.Pq, 78.55.- m, 78.67.De

The importance of the wetting layer (WL) in mediating cavity feeding was demonstrated experimentally by emptying the WL of carriers by using an applied electrical field [28] as well as by fabricating pyramidal QDs with negligible overlap between QD and WL states [29]. In both cases, large-detuning cavity feeding was suppressed.

Previously, a semiclassical Monte Carlo model with hybridization between QD and WL states, leading to a weak continuum-like background of available optical transitions that is subsequently Purcell enhanced [30], was used to explain nonresonant cavity feeding [9]. However, the semiclassical Monte Carlo model does not explain why the cavity feeding in highly detuned systems strongly favors positive detuning compared to negative and was only shown to account for detunings up to $7 \mathrm{meV}$ [9].

In this Letter, we present a full quantum mechanical model of strongly detuned systems that is able to describe the full bandwidth over which cavity feeding was experimentally observed, while at the same time providing a simple physical picture of the physical process mediating cavity feeding. We thus show that the coupling arises due to Coulomb-mediated transitions in the WL that provide energy to overcome the detuning between QD and cavity. Such Auger processes were also suggested in Ref. [31], but not analyzed quantitatively. To keep the physical picture simple, we limit our discussion to the $s$ shell of the QD and include neutral states up to two electron-hole pairs.

We consider a cQED model described by the second quantized system Hamiltonian,

$$
\mathcal{H}_{S}=\mathcal{H}_{0, e}+\mathcal{H}_{0, h}+\mathcal{H}_{\mathrm{cav}}+\mathcal{H}_{\mathrm{dip}}+\mathcal{H}_{C},
$$

with $\mathcal{H}_{0, \lambda}=\sum_{i} \hbar \omega_{i}^{\lambda} \hat{\lambda}_{i}^{\dagger} \hat{\lambda}_{i}$, with $\hat{\lambda}=\hat{e}, \hat{h}$ being the free QD part and $\hat{e}_{i}\left(\hat{h}_{i}\right)$ denoting annihilation of the $i$ th electron (hole) state with free energy $\hbar \omega_{e}\left(\hbar \omega_{h}\right)$. The cavity, 
including either 0 or 1 photon (no significant changes occurs by including more photons in the cavity description), is described by $\mathcal{H}_{\text {cav }}=\hbar \omega_{C} \hat{a}^{\dagger} \hat{a}$, where $\hat{a}$ is the photon annihilation operator. The light-matter coupling is given by $\mathcal{H}_{\text {dip }}=\sum_{i j} \hbar g_{i j}\left(\hat{e}_{i}^{\dagger} \hat{h}_{j}^{\dagger} \hat{a}+\hat{h}_{j} \hat{e}_{i} \hat{a}^{\dagger}\right)$, where $g_{i j}$ is the coupling constant, which is nonzero only if an optical transition exists between the two states. Finally, the Coulomb interaction in the QD is included through $\mathcal{H}_{C}=$ $\frac{1}{2} \sum_{i j k l} V_{i j k l}^{e e} \hat{e}_{i}^{\dagger} \hat{e}_{j}^{\dagger} \hat{e}_{k} \hat{e}_{l}+\frac{1}{2} \sum_{i j k l} V_{i j k l}^{h h} \hat{h}_{i}^{\dagger} \hat{h}_{j}^{\dagger} \hat{h}_{k} \hat{h}_{l}-\sum_{i j k l} V_{i j k l}^{h e} \times$ $\hat{h}_{i}^{\dagger} \hat{e}_{j}^{\dagger} \hat{e}_{k} \hat{h}_{l}$, where $V_{i j k l}^{\lambda, \lambda^{\prime}}$ is the Coulomb element connecting the different QD states as defined in standard textbooks on semiconductor optics [32].

The dynamics of the system is analyzed using a quantum master equation approach [33,34] for the reduced density matrix $\rho(t)$,

$$
\partial_{t} \rho(t)=-\mathrm{i} \hbar^{-1}\left[\mathcal{H}_{S}, \rho(t)\right]+\mathcal{L}(t)+S_{\mathrm{WL}}(t),
$$

where $\mathcal{L}(t)$ [35] represents losses through Lindblad terms [36]. This accounts for the decay of the excited QD states with rate $\Gamma$ due to spontaneous radiation into modes other than the cavity mode as well as nonradiative decay processes. Also, cavity decay with rate $\kappa$ and incoherent QD pumping with rate $P$ are included. Finally, $S_{\mathrm{WL}}(t)$ is the time-local WL reservoir-induced scattering term, introducing the dynamics of the non-Markovian reservoir [21,33]. For a more detailed description, see the Supplemental Material [37].

The QD system contains an $s$ shell, allowing spin up ( $\uparrow$ ) and spin down ( $\downarrow$ ) [38]. Only neutral and optically active states are included, and thus four different states are considered: $|e\rangle|h\rangle=|0\rangle|0\rangle,|\uparrow\rangle|\downarrow\rangle,|\downarrow\rangle|\uparrow\rangle,|\uparrow \downarrow\rangle|\uparrow \downarrow\rangle$. The QD pump rate and WL carrier concentration are assumed to be connected linearly according to Ref. [40].

Solving Eq. (2) numerically, we obtain the steady-state optical emission spectra shown in Fig. 1(a) [41]. It is noted that the choice of QD states results in an antibonding biexciton state with positive binding energy as discussed and investigated experimentally in Ref. [43]. Similar results are obtained using wave functions leading to the usual bonding biexciton state.

The presence of cavity feeding in the large detuning regime is clearly visible in Fig. 1 where both the emission spectra and the power dependence of the various peaks show excellent qualitative agreement with the experiments performed by Winger et al. [9]. The intensity of the exciton peak increases with pumping until saturation, and simultaneously the intensity of the cavity peak increases. Comparing calculations including and excluding the coupling to the WL, we conclude that the WL is responsible for the enhanced cavity feeding shown in Fig. 1.

We now turn to a more detailed discussion of the model and the Auger processes leading to the WL-mediated cavity feeding. The WL reservoir enters the dynamics via the Coulomb interaction through the scattering term $S_{\mathrm{WL}}(t)$
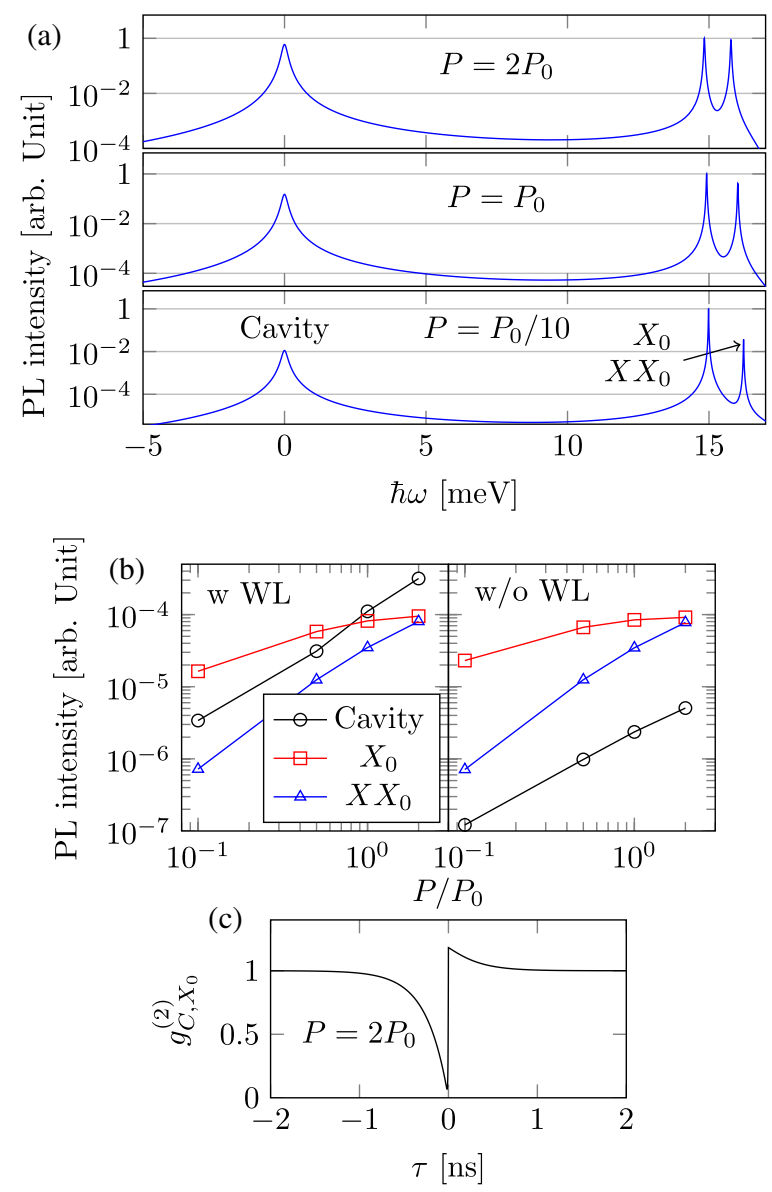

FIG. 1 (color online). (a) Steady-state cavity emission spectrum for different pumping rates for a cavity red detuned by $15 \mathrm{meV}(\hbar \Delta=15 \mathrm{meV})$ from the QD resonance. The reference pumping rate and concentration are taken as $\hbar P_{0}=0.5 \mu \mathrm{eV}$ and $n_{0}=2.5 \times 10^{15} \mathrm{~m}^{-2}$, respectively. The exciton (" $X_{0}$ "), biexciton (" $X X_{0}$ "), and cavity lines ("Cavity") are indicated. (b) Integrated emission of the different peaks with and without the WL interaction. (c) Normalized second-order cross correlation function $g_{C, X_{0}}^{(2)}(\tau) ; \tau>0$ implies observation of a photon in the exciton line after detection of a photon in the cavity line.

in Eq. (2). We consider a coupling Hamiltonian, between the system (S) and the reservoir (R), of the form $\mathcal{H}_{\mathrm{SR}}=$ $\sum_{\lambda} \sum_{\nu_{1} \nu_{2}} \hat{P}_{\nu_{1} \nu_{2}}^{\lambda} \hat{B}_{\nu_{1} \nu_{2}}^{\lambda}$, where $\hat{P}_{\nu_{1} \nu_{2}}^{\lambda}$ is a system operator and $\hat{B}_{\nu_{1} \nu_{2}}^{\lambda}$ is a reservoir operator that contains the QD-WL overlap elements [37]. Thus, $\hat{B}_{\nu_{1} \nu_{2}}^{\lambda}$ describes the interaction between the WL and the QD states $\left(\nu_{1}, \nu_{2}\right)$ for band $\lambda$. In this case, it can be shown [21] that the central quantity in $S_{\mathrm{WL}}(t)$ is the reservoir correlation function $D(t)$,

$$
D_{\nu_{1} \nu_{2} \nu_{1}^{\prime} \nu_{2}^{\prime}}^{\lambda}(t)=\left\langle\hat{B}_{\nu_{1} \nu_{2}}^{\lambda}(t) \hat{B}_{\nu_{1}^{\prime} \nu_{2}^{\prime}}^{\lambda}(0)\right\rangle_{R}
$$

where $\langle\cdot\rangle_{R}$ denotes a trace over reservoir states. For a more elaborate discussion of the general scattering term, see the Supplemental Material [37] and Ref. [21]. 
Including the WL-QD interaction introduces a continuum of WL states in the Coulomb Hamiltonian [44]. The Coulomb Hamiltonian can be split into three parts depending on the type of operators entering. (i) All operators are either QD or WL operators. This describes interactions solely in the QD or solely in the WL. The Coulomb interaction between QD states is included in $\mathcal{H}_{S}$ [Eq. (1)], and the many particle interactions between all carriers in the WL are treated within the random phase approximation $[32,37,46]$ by including screening of the Coulomb interaction. (ii) More QD operators than WL operators or vice versa. These terms do not conserve the number of particles in the QD and WL; thus, they describe either capture or escape processes. (iii) An Equal number of QD and WL operators. Here the particle number is conserved in both the QD and the WL. These terms are essential for describing cavity feeding.

To simplify the description, we only include the terms (iii). Thus, capture or escape processes are treated phenomenologically by assuming a fixed relation between the pumping rate and the WL carrier concentration. This leads to the coupling Hamiltonian [37]

$$
\begin{aligned}
\mathcal{H}_{\mathrm{SR}} & =\sum_{\nu_{1} \nu_{2}} \hat{e}_{\nu_{1}}^{\dagger} \hat{e}_{\nu_{2}} \hat{B}_{\nu_{1} \nu_{2}}^{e}+\sum_{\nu_{1} \nu_{2}} \hat{h}_{\nu_{1}}^{\dagger} \hat{h}_{\nu_{2}} \hat{B}_{\nu_{1} \nu_{2}}^{h}, \\
\hat{B}_{\nu_{1} \nu_{2}}^{\lambda} & =\sum_{\substack{k q \\
k \neq q}}\left[M_{\nu_{1} k q \nu_{2}}^{\lambda, e} \hat{e}_{\boldsymbol{k}}^{\dagger} \hat{e}_{\boldsymbol{q}}+M_{\nu_{1} k \boldsymbol{q} \nu_{2}}^{\lambda, h} \hat{h}_{\boldsymbol{k}}^{\dagger} \hat{h}_{\boldsymbol{q}}\right],
\end{aligned}
$$

where the elements $M_{\nu_{1} k q \nu_{2}}^{\lambda, \lambda^{\prime}}$, as defined in the Supplemental Material [37], contain the QD-WL Coulomb elements connecting the QD states $\left(\nu_{1}, \nu_{2}\right)$ and WL states $(\boldsymbol{k}, \boldsymbol{q})$.

Inserting Eq. (4) into Eq. (3) yields the reservoir correlation function $D(t)$. Two approximations are made for the WL reservoir: (i) The reservoir Hamiltonian contains only quadratic terms; (ii) the reservoir occupation is approximated by quasiequilibrium Fermi-Dirac distributions, appropriate for continuous excitation experiments.

The real part of the Fourier transform of $D(t)$ gives information about the interaction with the QD and can thus be considered as an effective reservoir spectrum [21]. As derived in detail in the Supplemental Material [37], we have

$$
\begin{aligned}
\operatorname{Re}\left\{D_{\nu_{1} \nu_{2} \nu_{1}^{\prime} \nu_{2}^{\prime}}^{\lambda, \lambda^{\prime}}(\hbar \omega)\right\}= & \pi \hbar \sum_{s=e, h} \sum_{\substack{k q \\
k \neq q}} M_{\nu_{1} k q \nu_{2}}^{\lambda, s} M_{\nu_{1}^{\prime} q k \nu_{2}^{\prime}}^{\lambda^{\prime}, s} n_{k}^{s} \\
& \times\left(1-n_{q}^{s}\right) \delta\left(\hbar \omega-\left[E_{q}^{s}-E_{k}^{s}\right]\right) .
\end{aligned}
$$

In the regime of large detuning, the light-matter interaction only weakly couples the bare QD and cavity states, which are separated by $\approx \hbar \Delta$. Here, the main role of the WL reservoir is to compensate the energy mismatch to allow the pumped QD to decay into a cavity photon. For this reason, we expect the WL scattering term to scale as $S_{\mathrm{WL}}(t) \propto D(\omega=\Delta)$ [37].
The structure of Eq. (5) describes a process as sketched in Fig. 2. The QD-cavity system emits $(\omega>0)$ or absorbs $(\omega<0)$ the excess energy from the recombination to or from the WL carriers. This scatters WL carriers around the Fermi edge to either higher or lower energies. The Auger process requires an occupied carrier state in the $\mathrm{WL}\left(n_{k}^{s}\right)$ to scatter to an unoccupied state $\left(1-n_{q}^{s}\right)$, where the energy difference between the two WL states matches the detuning. It is evident that such processes will enhance both emission and absorption of photons. As we are considering the large detuning limit, effectively placing the system in weak coupling, the energy flow will preferentially be from the QD to the cavity since reabsorption, even though enhanced, is highly unlikely due to the large detuning. The following discussions are thus concerned with the emission of photons from the QD.

In Fig. 3(a) the calculated spectral function $D_{\uparrow \uparrow \downarrow}^{h, h}(\hbar \omega)$ is shown for different temperatures. This spectral function describes the interaction between a spin up and a spin down QD hole and the WL carriers (both electrons and holes). Each spectral function contains several transition rates, even if corresponding to a diagonal transition in the pure system, because the system is dressed by both the cavity coupling and the Coulomb interaction. We include all possible combinations for the full calculations. Figure 3 thus shows a representative function.

To further investigate the effect of the WL coupling, we calculated the integrated peak intensities as a function of detuning; see Fig. 3(b). For large detunings, the cavity line contributes a large fraction of the total intensity. As the detuning increases and $D(\omega=\Delta)$ decreases, the intensity of the cavity peak also decreases. The detuning dependence of the integrated cavity intensity is seen to qualitatively match the energy dependence of the spectral function in Fig. 3(a).

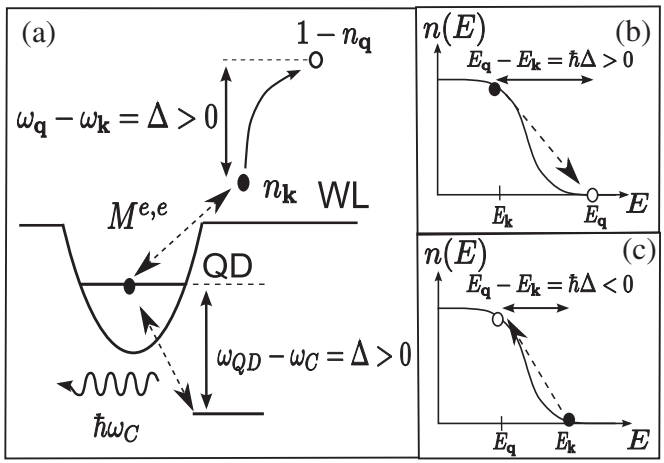

FIG. 2. (a) Auger process mediating photon nonresonant emission. The excess energy from the recombination process is supplied by the WL by scattering an electron from an occupied state to an unoccupied state in the WL. (b) Fermi-Dirac distribution with illustration of electron scattering for a scattering process assisting photon emission for red detuned cavities $(\Delta>0)$. (c) As in (b), but for blue detuned cavities $(\Delta<0)$. 

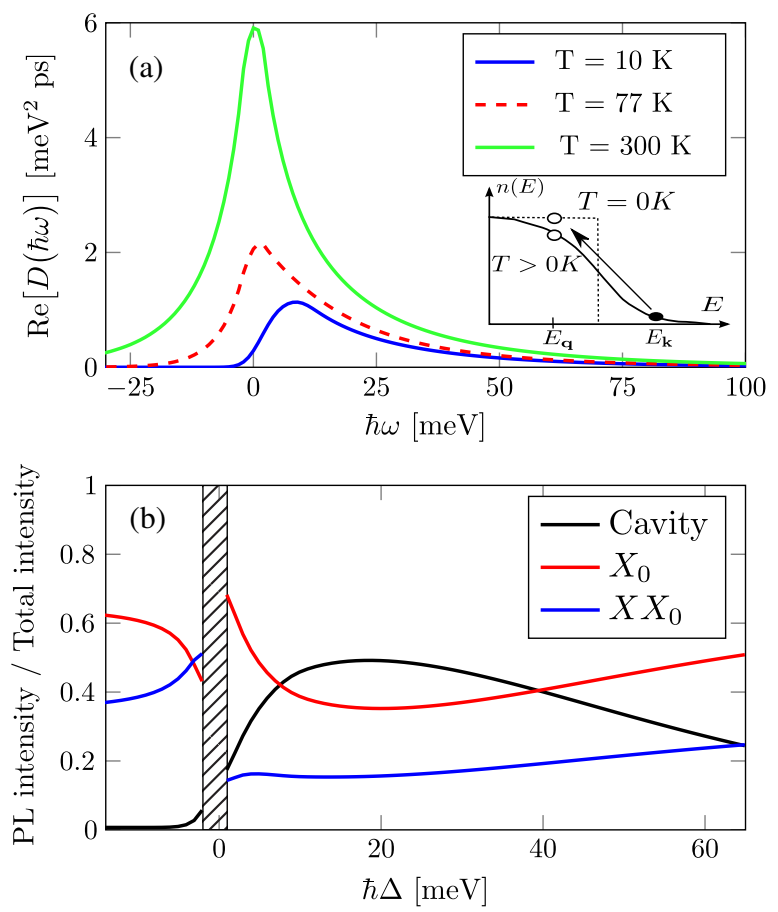

FIG. 3 (color online). (a) The spectral function $D_{\uparrow \uparrow \downarrow l}^{h, h}(\hbar \omega)$ for different temperatures and a carrier concentration of $n=2.5 \times$ $10^{15} \mathrm{~m}^{-2}$. The inset illustrates the Fermi-Dirac distribution at different temperatures. (b) The integrated peak intensities normalized to the total intensity as a function of the detuning for $P=P_{0}$ and $T=10 \mathrm{~K}$. The detunings leading to resonance with the cavity line have been omitted for clarity.

An important feature is the asymmetry between positive and negative detunings. This agrees with experimental observations, which find the cavity feeding to exist at larger absolute detunings when the cavity is redshifted $(\Delta>0)$ with respect to the emitter resonance, as compared to blueshifted cavities [9]. The asymmetry arises because the probability to promote up scattering in the WL is higher than that of down scattering due to the nature of the carrier distribution function. Thus, the experimentally observed asymmetries are a natural consequence of the Pauli principle as clearly displayed by our theory [Eq. (5)].

From Fig. 3(a) it is noticed that the model predicts cavity feeding to be enabled at larger absolute detunings for increasing temperature. This follows simply from the form of the Fermi-Dirac function, which flattens out with temperature, enabling scattering to lower energy states, as shown in the inset of Fig. 3(a). This temperature dependence has, to our knowledge, not been discussed previously in the literature.

The carrier concentration in the WL is another important parameter governing the coupling to the WL, as shown in the Supplemental Material [37]. The larger number of scattering possibilities in the WL for increased carrier concentrations is reflected by an increasing magnitude and shift of peak position of the spectral function. As the carriers participating in the Auger processes originate from states in the vicinity of the Fermi level, different WL states thus mediate the photon emission depending on the carrier concentration. WL states with large wave vectors are therefore responsible for the cavity feeding at larger carrier concentrations.

Finally, we have investigated the photon correlations by calculating the second-order cross correlation function, $g_{C, X_{0}}^{(2)}(\tau)$, cf. Fig. 1(c). This is a measure of the probability of observing a photon in the exciton line at $\tau$, when a photon in the cavity line is observed at $\tau=0$. The antibunching observed for $\tau<0$ reflects that measurement of an exciton photon at $-|\tau|$ leaves the system in the ground state, where repumping is needed before the cavity photon can be observed at $\tau=0$. The bunching peak seen for $\tau>0$ arises due to the cascaded nature of the biexcitonexciton system. The biexciton decays via emission of a cavity photon, assisted by Auger processes, leaving the system in the exciton state, which immediately decays. This agrees with the experimental data presented in Refs. [4,9]. Furthermore, the calculated correlation function shows better qualitative agreement with measurements for the sharp peak at $\tau=0^{+}$compared to the semiclassical model presented in [9].

It should be noted that the model does not show the experimentally observed photon bunching at $\tau=0$ in the cavity autocorrelation function [4,9]. This is well understood and reflects the fact that we only include the $s$ shell. The bunching peak at $\tau=0$ requires multiple independent decay channels for the cavity emission [9]. Since we have included only the $s$ shell, no such channels exist. If we include the $p$ and $d$ shells, we get several independent decay channels leading to a bunching peak for $\tau=0$.

To conclude, we have shown that Auger processes in the continuum of wetting layer states can mediate emission from an optical cavity, even when the cavity is detuned by tens of meV from the quantum dot that feeds it. This simple picture is derived from a comprehensive theory of the cavity quantum electron dynamics of the system, yet provides a simple picture that explains main experimental observations.

Financial support from Villum Fonden via the NATEC Center of Excellence is gratefully acknowledged. The Center for Nanostructured Graphene (CNG) is sponsored by the Danish Research Foundation.

*mikkel.settnes@gmail.com

[1] J. M. Gerard, B. Sermage, B. Gayral, B. Legrand, E. Costard, and V. Thierry-Mieg, Phys. Rev. Lett. 81, 1110 (1998).

[2] P. Michler, A. Kiraz, C. Becher, W. V. Schoenfeld, P. M. Petroff, L. Zhang, E. Hu, and A. Imamoglu, Science 290, 2282 (2000). 
[3] J. P. Reithmaier, G. Sek, A. Loffler, C. Hofmann, S. Kuhn, S. Reitzenstein, L. V. Keldysh, V. D. Kulakovskii, T. L. Reinecke, and A. Forchell, Nature (London) 432, 197 (2004).

[4] K. Hennessy, A. Badolato, M. Winger, D. Gerace, M. Atature, S. Gulde, S. Falt, E. L. Hu, and A. Imamoglu, Nature (London) 445, 896 (2007).

[5] M. Kaniber, A. Laucht, T. Hurlimann, M. Bichler, R. Meyer, M. C. Amann, and J. J. Finley, Phys. Rev. B 77, 073312 (2008).

[6] K. J. Vahala, Nature (London) 424, 839 (2003).

[7] E. Knill, R. Laflamme, and G. J. Milburn, Nature (London) 409, 46 (2001).

[8] A. Laucht, N. Hauke, J. M. Villas-Boas, F. Hofbauer, G. Bohm, M. Kaniber, and J. J. Finley, Phys. Rev. Lett. 103, 087405 (2009).

[9] M. Winger, T. Volz, G. Tarel, S. Portolan, A. Badolato, K. J. Hennessy, E. L. Hu, A. Beveratos, J. Finley, V. Savona, and A. Imamoglu, Phys. Rev. Lett. 103, 207403 (2009).

[10] J. Suffczynski, A. Dousse, K. Gauthron, A. Lemaitre, I. Sagnes, L. Lanco, J. Bloch, P. Voisin, and P. Senellart, Phys. Rev. Lett. 103, 027401 (2009).

[11] D. Press, S. Gotzinger, S. Reitzenstein, C. Hofmann, A. Loffler, M. Kamp, A. Forchel, and Y. Yamamoto, Phys. Rev. Lett. 98, 117402 (2007).

[12] S. Strauf, K. Hennessy, M. T. Rakher, Y.-S. Choi, A. Badolato, L. C. Andreani, E. L. Hu, P. M. Petroff, and D. Bouwmeester, Phys. Rev. Lett. 96, 127404 (2006).

[13] A. Laucht, N. Hauke, A. Neumann, T. Gunthner, F. Hofbauer, A. Mohtashami, K. Muller, G. Bohm, M. Bichler, M. Amann, M. Kaniber, and J. J. Finley, J. Appl. Phys. 109, 102404 (2011).

[14] A. Naesby, T. Suhr, P. T. Kristensen, and J. Mork, Phys. Rev. A 78, 045802 (2008).

[15] A. Auffeves, J. M. Gerard, and J. P. Poizat, Phys. Rev. A 79, 053838 (2009).

[16] M. Yamaguchi, T. Asano, and S. Noda, Opt. Express 16, 18067 (2008).

[17] B. Krummheuer, V. M. Axt, and T. Kuhn, Phys. Rev. B 65 , 195313 (2002).

[18] U. Hohenester, A. Laucht, M. Kaniber, N. Hauke, A. Neumann, A. Mohtashami, M. Seliger, M. Bichler, and J. J. Finley, Phys. Rev. B 80, 201311 (2009).

[19] U. Hohenester, Phys. Rev. B 81, 155303 (2010).

[20] P. Kaer, T. R. Nielsen, P. Lodahl, A.-P. Jauho, and J. Mork, Phys. Rev. Lett. 104, 157401 (2010).

[21] P. Kaer, T. R. Nielsen, P. Lodahl, A.-P. Jauho, and J. Mork, Phys. Rev. B 86, 085302 (2012).

[22] A. Majumdar, E. D. Kim, Y. Gong, M. Bajcsy, and J. Vučković, Phys. Rev. B 84, 085309 (2011).

[23] C. Roy and S. Hughes, Phys. Rev. Lett. 106, 247403 (2011).

[24] A. Nysteen, P. Kaer, and J. Mork, Phys. Rev. Lett. 110, 087401 (2013).

[25] H. Kurtze, J. Seebeck, P. Gartner, D. R. Yakovlev, D. Reuter, A.D. Wieck, M. Bayer, and F. Jahnke, Phys. Rev. B 80, 235319 (2009).
[26] E. A. Muljarov and R. Zimmermann, Phys. Rev. Lett. 98, 187401 (2007).

[27] M. Lorke, T. R. Nielsen, J. Seebeck, P. Gartner, and F. Jahnke, Phys. Rev. B 73, 085324 (2006).

[28] N. Chauvin, C. Zinoni, M. Francardi, A. Gerardino, L. Balet, B. Alloing, L. H. Li, and A. Fiore, Phys. Rev. B 80, 241306 (2009).

[29] M. Calic, P. Gallo, M. Felici, K. A. Atlasov, B. Dwir, A. Rudra, G. Biasiol, L. Sorba, G. Tarel, V. Savona, and E. Kapon, Phys. Rev. Lett. 106, 227402 (2011).

[30] E. M. Purcell, H. C. Torrey, and R. V. Pound, Phys. Rev. 69, 37 (1946).

[31] G. Tarel, V. Savona, M. Winger, T. Volz, and A. Imamoglu, in Quantum Optics with Semiconductor Nanostructures (Woodhead Publishing, Cambridge, 2012).

[32] H. Haug and S. W. Koch, Quantum Theory of the Optical and Electronic Properties of Semiconductors (World Scientific, Singapore, 1994).

[33] H.P. Breuer and F. Petruccione, The Theory of Open Quantum Systems (Oxford University Press, New York, 2003).

[34] H. Carmichael, An Open System Approach to Quantum Optics (Springer-Verlag, Berlin, 1993).

[35] $L(\hat{O}, \gamma)=\frac{\gamma}{2}\left(2 \hat{O} \rho(t) \hat{O}^{\dagger}-\hat{O}^{\dagger} \hat{O} \rho(t)-\rho(t) \hat{O}^{\dagger} \hat{O}\right) \quad$ where $\mathcal{L}(t)=\sum_{i} L\left(\hat{O}_{i}, \gamma_{i}\right)$. We include $L\left(\hat{e}_{\downarrow}^{\dagger} \hat{h}_{\uparrow}^{\dagger}, P\right), L\left(\hat{e}_{\uparrow}^{\dagger} \hat{h}_{\downarrow}^{\dagger}, P\right)$, $L(a, \kappa), L\left(\hat{e}_{\uparrow} \hat{h}_{\downarrow}, \Gamma\right)$ and $L\left(\hat{e}_{\downarrow} \hat{h}_{\uparrow}, \Gamma\right)$.

[36] G. Lindblad, Commun. Math. Phys. 48, 119 (1976).

[37] See the Supplemental Material at http://link.aps.org/ supplemental/10.1103/PhysRevLett.111.067403 for a detailed expression and derivation of the spectral function for the WL reservoir.

[38] QD wave functions according to Ref. [39]. A twodimensional harmonic oscillator potential, with oscillator lengths $L_{e}=7.5 \mathrm{~nm}$ and $L_{h}=5.5 \mathrm{~nm}\left(\hbar \omega_{e}=20.9 \mathrm{meV}\right.$ and $\hbar \omega_{h}=15.4 \mathrm{meV}$ ), for in-plane confinement, and an infinite potential well of width $a=4 \mathrm{~nm}$ in the perpendicular direction.

[39] P. Hawrylak, Phys. Rev. B 60, 5597 (1999).

[40] T. R. Nielsen, P. Gartner, and F. Jahnke, Phys. Rev. B 69, 235314 (2004).

[41] Utilizing quantum optical toolbox to Python [42], and parameters $m_{e}^{*}=0.065 m_{0}, m_{h}^{*}=0.17 m_{0}, \epsilon=13.6 \epsilon_{0}$, $\hbar g=100 \mu \mathrm{eV}, \hbar \kappa=200 \mu \mathrm{eV}$, and $\hbar \Gamma=1 \mu \mathrm{eV}$.

[42] J. Johansson, P. Nation, and F. Nori, Comput. Phys. Commun. 184, 1234 (2013).

[43] S. Rodt, A. Schliwa, R. Heitz, V. Türck, O. Stier, R. Sellin, M. Strassburg, U. Pohl, and D. Bimberg, Phys. Status Solidi B 234, 354 (2002).

[44] WL states is taken as orthogonal plane waves [40], although we notice that this does not have significant influence on the general effect [45].

[45] T. Markussen, P. Kristensen, B. Tromborg, T. W. Berg, and J. Mørk, Phys. Rev. B 74, 195342 (2006).

[46] U. Bockelmann and T. Egeler, Phys. Rev. B 46, 15574 (1992). 